\title{
Highlights from the 2020 AAPS 360 Annual Meeting
}

\author{
Vivian A. Gray ${ }^{1}$, Dorys Argelia Diaz ${ }^{2}$, Jennifer Dressman ${ }^{3}$, Yasuhiro Tsume $^{4}$, and Nikoletta Fotaki ${ }^{5}$ \\ ${ }^{1}$ V. A. Gray Consulting, Hockessin, DE, USA. \\ ${ }^{2}$ Global Product Development, Pfizer Inc, Groton, CT, USA. \\ ${ }^{3}$ Fraunhofer Institute of Translational Medicine and Pharmacology, Frankfurt, Germany. \\ ${ }^{4}$ Biopharm-Sterile \& Specialty Product, Merck \& Co., Rahway, NJ, USA. \\ ${ }^{5}$ Department of Pharmacy and Pharmacology, University of Bath, Bath, UK.
}

e-mail:vagray@rcn.com

\section{INTRODUCTION}

T he American Association of Pharmaceutical Scientists (AAPS) held its PharmSci 360 Annual Meeting and Exposition event, October 26November 5, 2020. PharmSci 360, a premier gathering of pharmaceutical scientists from around the world, was fully virtual.

\section{ADVANCES AND INNOVATION IN FORMULATION DEVELOPMENT}

On October 28th, there was a Rapid Fire, Prologue, and Symposium on "Advances and Innovation in Formulation Development." A summary of the talks that are pertinent to dissolution topics are provided.

\section{Rapid Fire}

Moderated by Mamta Kapoor (FDA), the first speaker was Heather Boyce (FDA), with a talk titled "Establishing Bioequivalence for 'Additional Strengths' of Oral Modified-Release Drug Products'. She described the FDA guidance, Bioequivalence Studies with Pharmacokinetic Endpoints for Drugs Submitted Under an ANDA, that includes recommendations outlining approaches for establishing bioequivalence of additional strengths of a proposed modified release (MR) drug product for oral administration. Through two case studies of Bupropion $\mathrm{HCl}$ extended-release (ER) tablets and Venlafaxine $\mathrm{HCl}$ ER tablets, she illustrated that the FDA's current thinking places less emphasis on compositional proportionality requirements than prior years. Whether a product is considered compositional or not, additional justification related to the proposed product release mechanism and excipient levels should be provided to use alternative methods to support and strengthen the bioequivalence study.

The second speaker was Sumit Arora (Certara), whose talk was titled, "Integrating Topical Drug Product Quality Attributes Within Physiologically-Based Pharmacokinetic Models." He began by describing physiologically based pharmacokinetic (PBPK) models, which are informed by prior knowledge of human anatomy, physiology, genetics, and the associated variability. PBPK modeling provides a robust framework to integrate physiochemical properties of a drug substance and formulation quality attributes, thereby enabling understanding of complex interplay of drug/drug products and human physiology. He went on to explain that application of these (semi)mechanistic models in the field of locally acting drug products is particularly interesting because drug concentrations at the local site of action (hardly accessible via traditional experimentation) can be related to the therapeutic performance. He discussed the considerations and key parameters needed to develop and verify/validate a mechanistic dermal absorption model (as implemented in Simcyp Simulator V19) capable of explaining observed in vitro and in vivo permeation of drugs across skin from topical drug products. He presented a Simkin case study discussing the application of PBPK model, verified with in vitro skin permeation, to predict in vivo exposure of topically applied drug products.

\section{Prologue}

Chris Moreton (Finnbrit Consulting) gave a talk titled, "Poor Solubility - Where do we stand 25 years after the "Rule of Five'". He started his presentation with some historical content, specifically what is the "Rule of 5" (Lipinski et al, J. Adv. Drug Devil Rev. 1997, 23, 3-25). In summary the rule of five is: 1 ) more than $5 \mathrm{H}$-bond donors; 2) molecular weight is over 500; 3) The $\log P>$ 5; and 4) more than $10 \mathrm{H}$-bond acceptors. The limitations are many but importantly it does not predict absorption or bioavailability. Today's combinational chemistry and high throughput screening gives the ability to optimize drug-receptor binding. What can we do to overcome poor water solubility? This can be done with increasing the effective surface area and concentration gradient. Also, self-emulsifying systems can assist in solubilizing the drug. He also pointed out that the choice of the dissolution test is critical; The test should be used to detect batches 
that would not give adequate drug release, raising the question that a quality control test may not be adequate for a poorly soluble drug.

\section{Symposium - High Drug-Loaded Amorphous Dispersions}

This symposium was focused on high drug-loaded amorphous dispersions (HDLAD). There were three speakers covering different aspects of HDLAD including processing in drug loading, improving the diffusivity and dissolution, and formulation of amorphous solid dispersions (ASDs).

The first speaker was Anura Indulkar (AbbVie), whose talk was titled, "Strategies to Formulate High DrugLoad Amorphous Dispersions to Reduce Pill-Burden." This talk provided an overview of ASDs, followed by a description of their dissolution behavior. Strategies to increase drug loading without compromising dissolution were discussed, including exploiting ionization behavior of the drug to enhance drug loading in the ASD and incorporating additives to improve dissolution of ASDs. There were three ways to enhance drug loading in ASDs: 1) polymers, 2) surfactants, and 3) ionization.

Yinshan Chen (Genentech) spoke next on the topic, "Counterions Facilitate High Drug Loading in Amorphous Solid Dispersion." High drug loading of ASDs challenges physical stability and drug release. In her research, counterions were incorporated with an active pharmaceutical ingredient (API) model compound, indomethacin, to ionize the API and influence the $\mathrm{pH}$ of the diffusion layer. Improvement of the dissolution rate was systemically studied to show the facilitating effect of counterions in high drug load ASDs. These findings could help design better HDLADs using counterions for ionizable drugs.

The last speaker was Deana Mudie (Lonza Global Research and Development). Her talk was tilted, "A Novel Architecture for High Drug-loaded Amorphous Solid Dispersion Tablets; Assessment of In Vitro and In Vivo Performance, Stability and Manufacturability." She provided a high-loaded dosage form (HLDF) architecture for ASDs that was developed using a spray-dried high-glass transition temperature dispersion polymer to facilitate high drug loading while maintaining physical stability. The ASD was then granulated with concentrationsustaining polymers to extend supersaturation in solution. She discussed case studies highlighting the HLDF architecture, including physical stability, in vitro and in vivo performance, and manufacturability.

\section{PARTNER PRESENTATION: DEVELOPING MODIFIED RELEASE VERSIONS OF}

IMMEDIATE RELEASE SOLID DOSAGE FORMS On October 29, Richard Sidwell (RECRO) and Wayne Wiley (RECRO) gave a join presentation on the topic, "Regulatory and CMC Considerations for Developing Modified Release Versions of Immediate Release Oral Solid Dosage Forms."

They began with stating that modified-release formulations offer patient compliance, marketing, and exclusivity/patent benefits over their immediaterelease predecessors. However, modified-release dosage forms can also present their own unique set of complications, nuances, and regulatory expectations. They shared examples and perspectives from decades of industry experience. They provided insight into strategic decisions, explored key data to acquire during API characterization and preformulation, and shared health authority expectations for modified-release formulations compared to existing immediate-release dosage forms. They discussed the dissolution test as an important part of the dosage form development and approval process. An in vitro-in vivo correlation (IVIVC) is expected, although it may be not the regulatory method for routine use. They emphasized that discussion of CMC issues, especially the dissolution method, should be a part of the usual "granted" preapproval meeting with FDA, including the pre-IND (investigational new drug), EOP2 (end of phase 2), and pre-NDA (new drug application) meeting.

\section{HOT TOPIC - "ROLE OF MODELING AND SIMULATION IN BIOPHARMACEUTICS"}

This hot topic session was held on November 3, 2020. It was moderated by Mong-Jen Cheng (AbbVie) with three speakers who presented a 15-minute talks followed by question and answer session. The hot topic session discussed the use of physiologically based pharmacokinetic and biopharmaceutics (PBPK/PBBM) modeling and factors to consider when undertaking a PBPK modeling.

The first talk was given by Patrick J. Marroum (AbbVie), who presented the role of modeling and simulation in biopharmaceutics. He discussed in detail the challenges in modeling and simulations and shared prerequisites for establishment of successful modeling. Patrick highlighted that modeling and simulation are not new concepts, because the first regulatory guidance document addressing the development, evaluation, and application of IVIVC was issued in 1997. The advancement of PBPK/ PBBM modeling software and its availability to increase 
industry and regulatory acceptance for critical product performance decision making has contributed to a wide use of virtual $B E$ simulations to assess the impact of in vitro release between two drug products. The advantages and disadvantages of empirical and mechanistic PBPK models were reviewed. The following aspects were emphasized as a prerequisite to establish a successful model: a crossmultidisciplinary collaboration; a sensitive and discriminating dissolution method; adequate planning to design the appropriate experiments; in vivo and in vitro data availability from formulation variants with various release characteristics; and global reception of the models and their application. Also discussed were the value and application of modeling in formulation selection, clinically relevant dissolution specifications, establishment of safe space, in vivo bioavailability and bioequivalence waiver, and virtual BE. There has been increased reliance on modeling and simulations both from the industry and regulatory perspectives, resulting in a need to globally harmonize the regulatory expectations and standards of modeling and simulations.

Next, Dwaipayan Mukherjee (AbbVie) presented "Modeling Approaches." Dwaipayan provided an overview of model-based approaches for predicting in vivo impact of biopharmaceutics aspects and factors that need to be considered when designing a PBPK model for drug substances. He provided some literature-based examples of PBPK/PBBM model-based approaches for IVIVC, safe space and dissolution specifications, and virtual $B E$. He also shared three different methods to incorporate in vitro dissolution data into PBPK models and explained their advantages and disadvantages. The following methods were explained in detail: input directly as dissolution kinetics (in-vitro data from USP apparatus, less mechanistic), input as release kinetics (considers $\mathrm{pH}$-dependent dissolution in gastrointestinal [GI] track), and input via dissolution fitting module (more mechanistic, considers solubility, particle size). A case study following current FDA and European Medicines Agency (EMA) guidance for PBPK method development, model verification, and model application was presented. The PBPK model was used to assess the impact of wider dissolution specifications than what was originally proposed and to successfully justify the amendment of the approved commercial dissolution specification for commercial Eaglin $200 \mathrm{mg}$ tablets. The FDA regulatory agreed that the PBPK model was able to demonstrate that widening the specifications as requested by the applicant would not impact the clinical performance of the commercial tablets. The takeaways from this presentation are three-fold. First, PBPK models need to be independently developed and verified using clinical data. Second, it is imperative to have a solid understanding of the dissolution and absorption model including the required assumptions. Third, use of the PBPK model can result in greater regulatory flexibility and can potentially reduce the number of clinical studies needed to approve and maintain a product on the market.

The third talk was given by Banu Zolnik (Division of Biopharmaceutics, ONDP, OPQ, CDER, FDA) on "FDA's Perspective on the Physiologically Based Pharmacokinetic (PBPK) Analyses for Biopharmaceutics Application". Banu provided an overview of the purpose of PBPK modeling, which is to link the in vitro dissolution or other in vitro testing inputs to in vivo drug exposure. She highlighted the current regulatory landscape and mentioned the draft FDA guidance "The Use of Physiologically Based Pharmacokinetic Analyses-Biopharmaceutics Applications for Oral Drug Product Development, Manufacturing Changes, and Controls" issued in October 2020. She explained the general workflow of PBPK models included in the draft guidance and general strategy on developing PBPK modeling. Likewise, she discussed the type of information the PBPK study report should include for biopharmaceutics applications for regulatory submissions. The objective of the model should be clearly specified. Some examples include dissolution (development of a clinically relevant specification method and acceptance criteria) and other specifications (particle size, polymorphic form), bridging (clinical batches to the potentially marketed commercial product), and biowaiver studies for post-approval change. Flow charts include model development, verification, and validation. The model development and verification assumptions should be clearly presented as well as the rationale and supportive information on model parameters including formulation and in vitro dissolution. The clinical data should include the clinical study design, dosing regime, and study population. System data should include anatomical structured and physiological parameters for the GI track. Drug data should include solubility, permeability, ADME (absorption, distribution, metabolism, and excretion), particle size solid form and dissolution.

Key considerations to model validation are that acceptance criteria should be established a priori, and independent datasets (i.e., not used in model development) are recommended. Applicants are encouraged to use virtual $B E$ studies to predict the outcome. A description of intra- and inter-subject variability and justification of the number of subjects and trials used in virtual BE studies should be included. A 
case example of a PBPK model application was discussed to illustrate how PBPK modeling and simulation was used to support the selection of drug substance particle size distribution, set specifications based on parameter sensitivity analysis and virtual $\mathrm{BE}$.

The takeaways of this presentation were that the U.S. FDA supports innovative and data-driven mechanistic modeling approaches, and early interactions with the FDA on the use of modeling approaches is encouraged. The PBPK model acceptance criteria are not currently specified in the draft guidance, and the applicant is encouraged to propose the acceptance criteria based on the risk and intended purpose of the model.

\section{SYMPOSIUM: FORMULATION DEVELOPMENT CHALLENGES FOR EMERGING MODALITIES}

On November 4, this symposium, consisted of three topics: in vitro dissolution setting for oral drugs based on the human $\mathrm{Gl}$ physiology, prediction of in vivo bioperformance for oral drugs by in vitro dissolution and simulation/modeling, and PBPK modeling based on biorelevant data for pediatric patients.

The first speaker was Yasuhiro Tsume (Merck) who spoke on the topic, "GI Physiology and Condition to In Vitro Dissolution Methodologies as Intro". He discussed why compendial dissolution apparatus may not be able to predict in vivo dissolution profiles for certain drug products. He also emphasized which experimental conditions should be focused on and incorporated into the in vitro dissolution test when predicting in vivo bioperformance of oral drugs. He discussed important experimental factors such as gastric emptying, buffer $\mathrm{pH}$, volume, etc. by displaying the in vivo predictive dissolution profiles of ibuprofen and ketoconazole as example drugs. He also introduced in vivo predictive methodologies, TNO-TIM, ASD/GIS- $\alpha$ (transfer models), IDAS (absorption systems)/D-P (dissolution-permeation) systems, MacroFLUX and MicroFLUX, USP, and biphasic systems, and their pros and cons as well as their highthroughput screenability.

Jennifer Dressman (Fraunhofer Institute of Translational Medicine and Pharmacology) gave a talk on "In Vivo Predictive in Vitro Dissolution Methodologies (Current and Newer Methods)." Anticipating changes in the pharmacokinetics of new drugs is one of the key activities in the development of formulations for oral administration. In particular, it is important for the design of clinical studies to know and understand what effect taking the drug with a meal will have on the bioavailability of the drug. With the advent of the widespread use of acid reducing agents (ARA) like proton pump inhibitors, it is also important to understand if patients taking these medicines are able to absorb the drug to the same extent as those who do not. Lastly, it is of vital interest to formulators to be able to predict if the absorption of the drug can be enhanced by using so-called, "enabling" formulations. Dr. Dressman proposed using an in vitro-in silico approach to address all three of these questions.

Aprepitant was used to illustrate how the in vitro-in silico approach can be used to explain the modest food effect observed for this drug when it is formulated as the nanosized API. Data required for the approach include a) a reliable model for the post-absorptive pharmacokinetics of the API; b) solubility data for the drug under fasted and fed state conditions; c) dissolution data for the drug product under fasted and fed state conditions; d) reliable permeability data for the API; and e) two-stage testing to reveal the potential for API precipitation (for weak bases / enabling formulations like nanosized aprepitant). By combining biorelevant in vitro testing with PBPK modeling, the pharmacokinetics of aprepitant could be simulated under both fasted and fed conditions at two different doses. The game-changer with this approach is that, once the in vitro-in silico model has been verified, it is possible to explore the potential effects of variations in physiological parameters like gastric emptying, permeability, or intestinal $\mathrm{pH}$ on the overall pharmacokinetic profile in advance of clinical trials, thus optimizing clinical trial design.

Another application of the in vitro-in silico approach is to set dissolution specifications for batch release. Using zolpidem as a case example, the approach was first used to better understand the negative food effect of this BCS class I drug. Parameter sensitivity analysis suggested that the decreased rate of gastric emptying associated with intake of a rich meal is one of the main drivers of the negative food effect. The model was then used to explore the effect of dissolution on the plasma profile. It was concluded that, as long as the tablets dissolve within 45 minutes of intake, the plasma profile would not be detrimentally affected.

Next, a set of dissolution media representing gastric conditions after intake of ARA therapy was developed to forecast changes in bioavailability when the drug is given concomitantly to ARA therapy. By combining dissolution results in these media with PBPK modeling, it was possible to bracket the plasma profiles of a weakly basic drug when 
administered with ARA therapy.

Last, but not least, the ability of the in vitro-in silico approach to anticipate formulation effects on the plasma profile was illustrated using two non-bioequivalent formulations of raltegravir. Using the approach shown in the figure below, the differences between the plasma profiles of the suspension and tablet dosage forms of raltegravir were simulated well.

In conclusion, the in vitro-in silico approach offers an efficient way forward to evaluating various scenarios in the development of oral drug formulations.

The last speaker, Nikoletta Fotaki (University of Bath) closed the symposium with a talk on "Predicting Performance in Pediatrics with PBPK Modelling and Biorelevant Data." Biopharmaceutics tools in terms of biorelevant in vitro techniques and biopharmaceutics in silico techniques can support and facilitate pediatric pharmaceutical development. She described the development of pediatric biorelevant media simulating the $\mathrm{Gl}$ conditions in different pediatric age groups based on the biorelevant media developed for adults and taking into account the physiological conditions in the pediatric population. Solubility studies of a wide range of compounds in the pediatric biorelevant media revealed that developmental differences between pediatrics and adults translate to alterations of solubility.

Two case studies were presented. In the first case study, it was shown how the combination of biorelevant solubility and dissolution data with PBPK modeling led to successful prediction of the in vivo performance of an immediaterelease formulation of a BCS class II compound for the pediatric population. In the second case study, she discussed how biorelevant data and PBPK modeling can be used for the prediction of the effect of co-administration of food with granules in infants. She concluded her talk by emphasizing that successful biopharmaceutics tools for pediatric populations require reliable clinical experimental data coupled with mechanistic understanding of all ADME processes.

\section{SUMMARY}

This conference, which was completely virtual, hit on many topics relevant to dissolution testing. The experience of the attendees was positive. Although the inperson networking was absent, real-time questions from participants were useful, and having the convenience of being able to go back and replay the presentations was a plus. There is a trend on the use and application of modeling and simulation tools to assess product performance. There was an emphasis on the importance of interdisciplinary collaboration, continuous learning around modeling applications, and knowledge sharing on the applications of in vivo predictive methodologies in drug development.

\section{CONFLICT OF INTEREST}

The authors disclosed no conflicts of interest related to this article.

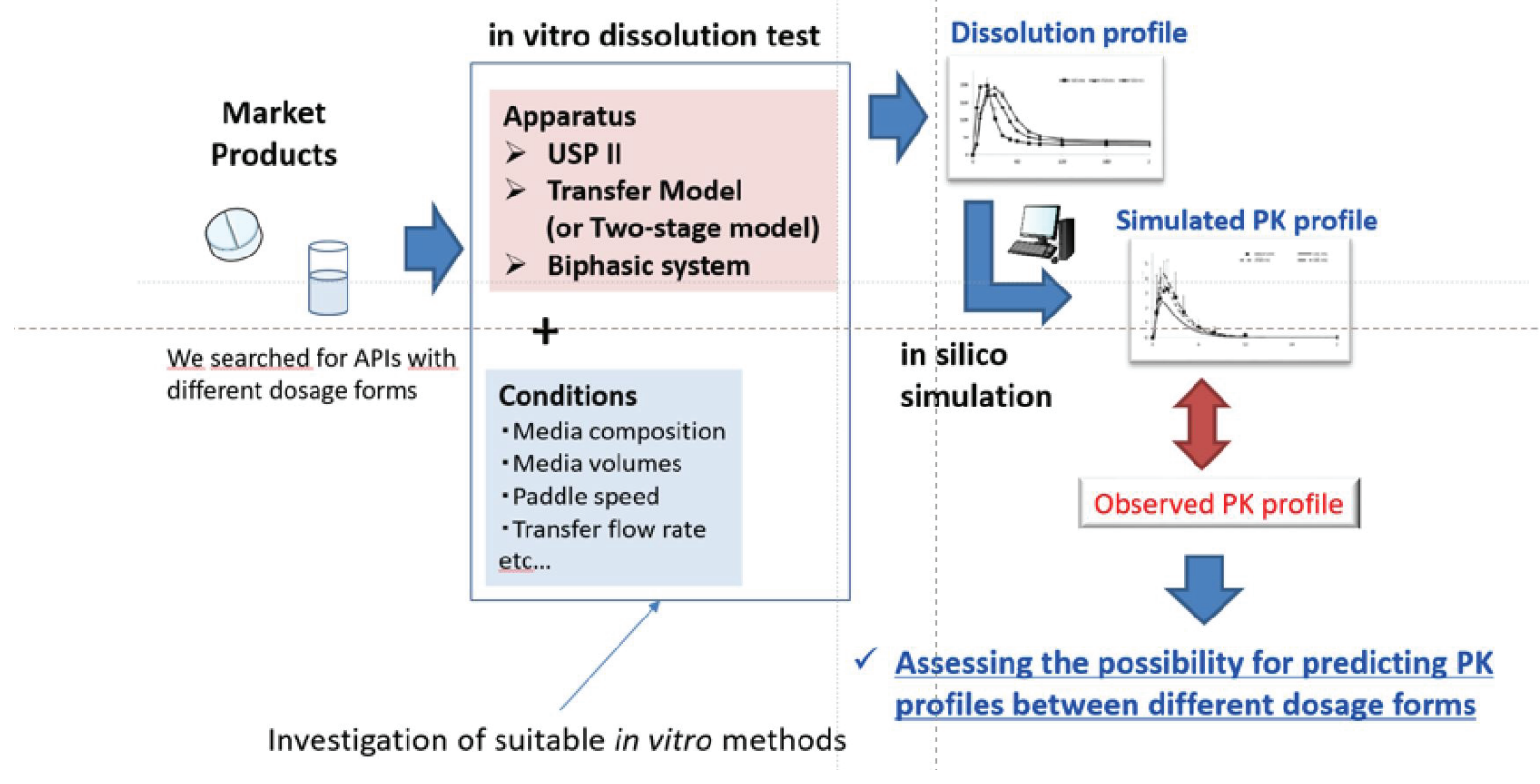

